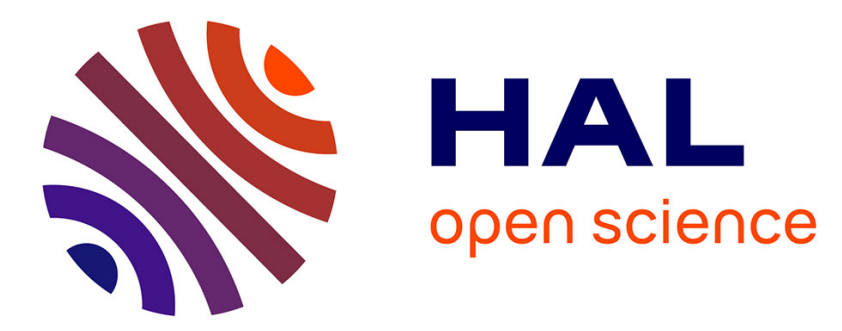

\title{
Time Domain Measurements for a Time Reversal SIMO System in Reverberation Chamber and in an Indoor Environment
}

\author{
Ijaz Haider Naqvi, Ghaïs El Zein
}

\section{To cite this version:}

Ijaz Haider Naqvi, Ghaïs El Zein. Time Domain Measurements for a Time Reversal SIMO System in Reverberation Chamber and in an Indoor Environment. 8th IEEE International Conference on Ultra-Wideband (ICUWB 2008), Sep 2008, Hanovre, Germany. pp.211-214, 10.1109/ICUWB.2008.4653388 . hal-00448873

\section{HAL Id: hal-00448873 \\ https://hal.science/hal-00448873}

Submitted on 20 Jan 2010

HAL is a multi-disciplinary open access archive for the deposit and dissemination of scientific research documents, whether they are published or not. The documents may come from teaching and research institutions in France or abroad, or from public or private research centers.
L'archive ouverte pluridisciplinaire HAL, est destinée au dépôt et à la diffusion de documents scientifiques de niveau recherche, publiés ou non, émanant des établissements d'enseignement et de recherche français ou étrangers, des laboratoires publics ou privés. 


\title{
Time Domain Measurements for a Time Reversal SIMO System in Reverberation Chamber and in an Indoor Environment
}

\author{
I. H. Naqvi, G. El Zein \\ Institute of Electronics and Telecommunication Rennes, IETR-UMR CNRS 6164 \\ INSA, 20 Avenue des Buttes de Coesmes, 35043, Rennes France. \\ ${ }^{1}$ Ijaz-Haider.Naqvi@ens.insa-rennes.fr \\ ${ }^{2}$ Ghais.El-Zein@insa-rennes.fr
}

\begin{abstract}
Time domain measurements are conducted for ultrawideband (UWB) signals in a reverberation chamber (RC) and in a typical indoor environment for a single input multiple output (SIMO) time reversal (TR) system. Channel estimation is carried out using an Arbitrary Waveform Generator (AWG) and a high speed Digital Storage Oscilloscope (DSO) for all transmitter receiver links. The measured channel impulse responses (CIR) of all the links are added and re-transmitted from the transmitting antenna. Upon reaching the receiving antennas, the received signals are added to form the eventual received signal. Different TR characteristics i.e. TR peak performance, TR focusing gain, average power increase, signal to side lobe ratio (SSR) and RMS delay spread are analyzed and compared to that of a single input single output (SISO) TR system.
\end{abstract}

Index Terms - Time reversal (TR), ultra-wideband (UWB), focusing gain, TR peak performance.

\section{INTRODUCTION}

Pulsed Ultra-wide band (UWB) is a communication technique in which high data rate can be achieved by making use of ultra large bandwidth. The ability of pulsed UWB to resolve individual multipath components is exploited in the recent research for short range communication applications. However, the large number of resolvable paths and the low power limitations necessitate a complex receiver system. To collect the received signal energy, Rake receiver, transmitreference or differential scheme and the decision feedback autocorrelation receiver can be implied [1]-[3]. Each technique has its own difficulties and drawbacks. The characteristics of an Impulse radio and use of Rake receivers for signal detection has already been studied for communication in dense multipath environment [4],[5]. Time reversal (TR) has been proposed as a technique to shift the design complexity from the receiver to the transmitter. Classically, TR has been applied in acoustics and under water communication applications [6][7] but in recent times, it has been widely studied for UWB communication. In a single input single output (SISO) TR scheme, the time-reversed channel impulse response (CIR) is used as a transmitter pre-filter. It results in a received signal which is considerably focused both in spatial and temporal domains. Owning to temporal focusing, the received power is concentrated within a few taps and the effects of inter symbol interference (ISI) are greatly reduced. The task of equalizer design becomes much simpler than without focusing. The receiver system becomes simpler and significant signal energy can be collected using simple energy threshold detectors [8]. Another important advantage of TR is the spatial focusing. It enables very low co channel interference in a multi cell system, resulting in a very effective use of bandwidth in the overall network. It is because of these characteristics that TR is gaining more and more attention for communication in UWB [9]-[18].

In [16], the authors have presented the first time domain measurements for an indoor environment. In this paper the, to the best of the authors knowledge, first ever time domain SIMO measurements are presented in reverberation chamber (RC) and in an indoor environment. A very narrow pulse is transmitted from an antenna toward multiple receiving antennas. The CIRs for one transmitting antenna and multiple receive antennas is measured and added together. The resulting signal is reversed in time and retransmitted from the same transmitting antenna and toward the same set of multiple antennas. The received signals from multiple antennas are again added together. TR characteristics like, focusing gain, peak to side lobe ratio, delay spread and average power augmentation are assessed. It is shown that with SIMO configuration a focusing gain of the order of $40 \mathrm{~dB}$ is achieved in a $\mathrm{RC}$ environment.

The rest of the paper is organized as follows. First, a brief introduction of TR is presented in section II. Experimental measurement setup and results are presented and analyzed in Section III. Finally, Section IV concludes this paper.

\section{TIME REVERSAL}

TR is essentially a pre-Rake scheme in which time reversed channel impulse responses (CIR) are used as transmitter prefilter. The signal (after being pre-filtered) propagates in an invariant channel following the same paths and results in coherently adding all the received signals in the delay and spatial domains. With this technique, a focusing gain in the 
order of $8 \mathrm{~dB}$ for indoor propagation channel, strong temporal compression and spatial focusing (depending on the signal band width) are observed [10]. The received signal quality is improved by the focusing gain, ISI effects are mitigated by temporal compression and multiuser interference is reduced due to spatial focusing. The received signal at the intended receiver $(j)$ can be mathematically represented as:

$$
s(t) \star h_{i j}(-t)^{*} \star h_{i j}(t)=s(t) \star R_{i j}^{a u t o}(t)
$$

where $h_{i j}(t)$ is the CIR from the transmitting point to an intended receiver, $s(t)$ is the transmitted signal, $\star$ denotes convolution product and $(.)^{*}$ means the complex conjugate of the function and $R_{i j}^{a u t o}(t)$ is the autocorrelation of the CIR between the transmitting antenna $(i)$ and receiving antenna $(j)$. The received signal at any non intended receiver $(k)$ is:

$$
s(t) \star h_{i j}(-t)^{*} \star h_{i k}(t)=s(t) \star R_{i k j}^{c r o s s}(t)
$$

where $h_{i k}(t)$ is the CIR from the transmitting point to an unintended receiver and $R_{i k j}^{\text {cross }}(t)$ is the cross-correlation of the CIR $h_{i k}(t)$ and the time reversed complex conjugated version of the transmitted signal $h_{i j}(-t)^{*}$. If the channels are uncorrelated, then the signal transmitted for one receiver will act as a noise for a receiver at any other location. This results in a secure communication with low probability of detect and low probability of intercept. If there are $N_{r}$ receiving antennas and one transmitting antenna, the received signal by the $j t h$ receiving antenna is:

$$
\begin{gathered}
y_{j}(t)=\underbrace{s_{j}(t) \star R_{i j}^{\text {auto }}(t)}_{\text {Signal }(j)}+\underbrace{\sum_{k=1 ; k \neq j}^{N_{r}} s_{k}(t) \star R_{i k j}^{\text {cross }}(t)}_{\text {Interference }(j)} \\
+\underbrace{n_{j}(t)}_{\text {Noise }(j)}
\end{gathered}
$$

where $s_{j}(t)$ and $s_{k}(t)$ are the transmitted signals intended for the $j t h$ user and the $k t h$ user respectively. If the channels are uncorrelated, the Interference part in (3) will be negligible, enabling an interference free communication with different users.

To assess the performance of a SIMO TR system, some TR characteristics are defined. TR peak performance improvement of a SIMO system is defined as the ratio of a TR received peak with SIMO system to the TR received peak with SISO system. Focusing gain (FG) of a TR system is defined as the ratio of the strongest tap power of the received signal in TR scheme to the strongest tap power of the pulse system:

$$
F G=20 \log _{10}\left(\frac{\max \left|y_{j}(t)\right|}{\max \left|h_{i j}(t)\right|}\right)
$$

The average received power with the TR scheme increases as compared to the pulsed system for the same value of the total transmitted energy. In this paper the comparison of the average power increase of the SIMO and SISO TR systems is made.

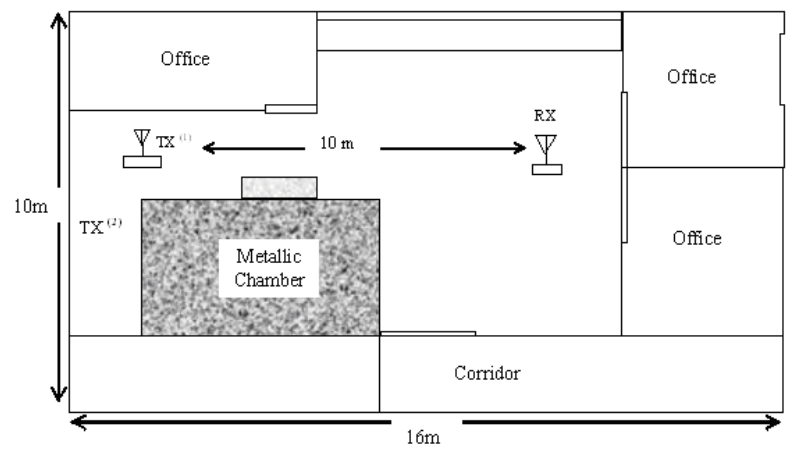

Fig. 1. Measurement environment layout

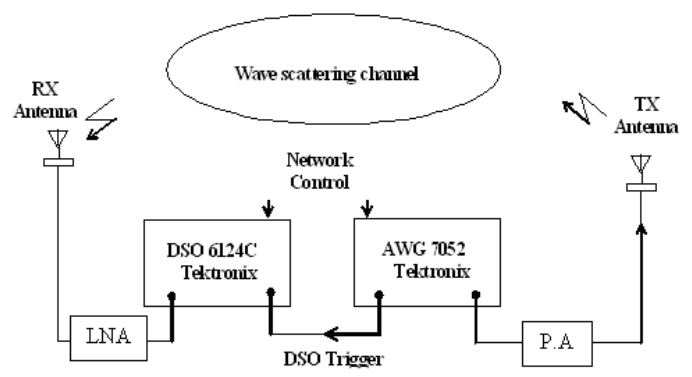

Fig. 2. Measurement setup

Signal to side lobe ratio (SSR) is defined as ratio of the power of the first to second strongest tap in a TR received signal:

$$
S S R=20 \log _{10}\left(\frac{\max \left|y_{j}(t)\right|}{\max \left|y_{j}(t)\right|_{t \neq t_{\text {peak }}}}\right)
$$

where $t_{\text {peak }}$ is the time for strongest tap. Another important TR characteristic is the instantaneous RMS delay spread $\left(\sigma_{t}\right.$ ). It can be calculated by the first and the second moment of the measured CIR or the measured TR response:

$$
\sigma_{\tau}=\sqrt{\frac{\sum_{l=1}^{N} P D P(l) \tau_{l}^{2}}{\sum_{l=1}^{N} P D P(l)}-\left(\frac{\sum_{l=1}^{N} P D P(l) \tau_{l}}{\sum_{l=1}^{N} P D P(l)}\right)^{2}}
$$

where $\operatorname{PDP}(l)=\left|h_{i j}(l)\right|^{2}, \tau_{l}$ is the excess time delay and $\mathrm{N}$ is the total length of the PDP. RMS delay spread is considered a metric for temporal compression in TR systems. In this paper we study the RMS delay spread ratio of a pulse UWB system and a TR UWB system. The delay spread ratio $\left(d s / d s_{T R}\right)$ must be as high as possible to have good temporal compression where $d s$ and $d s_{T R}$ are the RMS delay spreads of a pulse UWB system and a TR UWB communication system respectively.

\section{Experimental SETUP AND Simulation Results}

Experiments are performed in RC and typical indoor environments. $\mathrm{RC}$ is a metallic chamber of dimensions $8.7 \mathrm{~m} \times$ $3.7 m \times 2.9 m$ present inside IETR laboratory whose layout is shown in Fig. 1. For an indoor channel, an experimental 
(a)

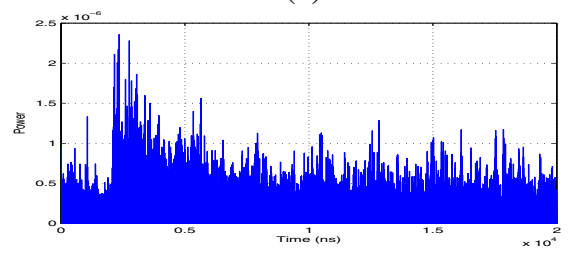

(b)

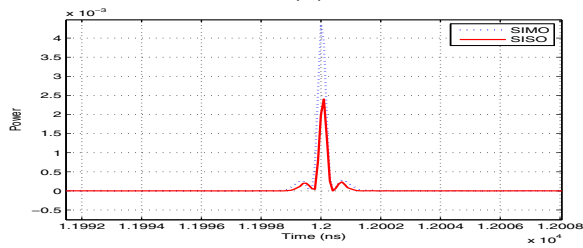

Fig. 3. In reverberation chamber a) SISO CIR b) TR response for SISO and SIMO configurations

TABLE I

TIME REVERSAL CHARACTERISTICS IN RC

\begin{tabular}{ccc}
\hline \hline TR Property & SISO TR & SIMO TR \\
\hline \hline Focusing Gain $(\mathrm{dB})$ & 27.7 & 28.59 \\
Avg. Increased Power & 3.26 & 4.98 \\
SSR & 10.56 & 10.92 \\
Normalized TR peak & 0 & 4.84 \\
\hline
\end{tabular}

setup is established in a modern laboratory building in IETR having the plan shown in Fig. 1. The indoor environment is an office space of $14 \mathrm{~m} \times 8 \mathrm{~m}$. All rooms are furnished with office equipments: tables, PCs and seats. RC increases the wave reflections in the environment and allows accomplishing a non line-of-sight (NLOS) propagation scenario. Measurement setup is illustrated in Fig. 2. One log periodic antenna is used as a transmitter and two conical mono-pole antennas are used as the receiver. The height of the transmitting antenna is $1.5 \mathrm{~m}$ from the ground and that of receiving antennas is $1 \mathrm{~m}$ from the ground. The distance between the transmit and receive antennas is $4.5 \mathrm{~m}$ and $9 \mathrm{~m}$ in $\mathrm{RC}$ and in indoor respectively. The channel sounding pulse and the time reversed CIR are generated through the arbitrary waveform generator (AWG 7052) having a maximum sampling rate of $5 \mathrm{GS} / \mathrm{s}$. The output signal of AWG is amplified to a level to drive the power amplifier (P.A), Mini-Circuits ZHL-42. The given P.A provides a constant gain of $30 \mathrm{~dB}$ and covers the frequency range of $700-4200 \mathrm{MHz}$. The output power is limited to avoid the interference to unauthorized bands and to generate sufficient signal-to-noise ratio (SNR) at the receiver. The receiver is a Digital Storage Oscilloscope (Tektronix DSO 6124C) with band width of $12 \mathrm{G} \mathrm{Hz}$ and a maximum sampling rate of 40 GS/s. DSO captures the CIR of the channel as well as the TR response. DSO is operated in average mode so that 8 samples are taken and averaged together. A set of 100 measurements is taken in RC and a set of 1000 measurements is taken in the indoor environment. Thus the results presented in this paper are average of 100 and 1000 measurements in RC and the indoor environment. (a)

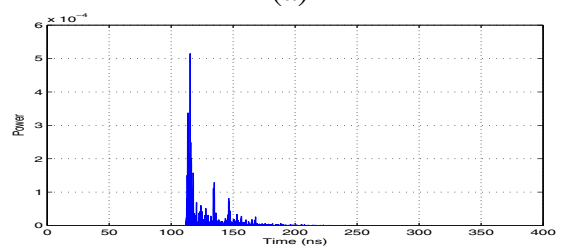

(b)

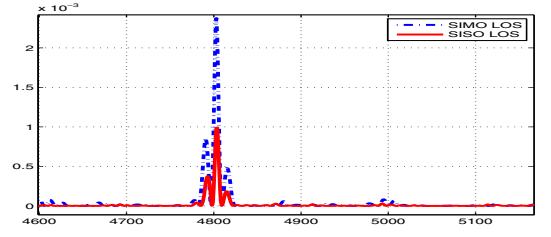

Fig. 4. In LOS configuration a) Indoor CIR for SISO b) Indoor TR response for SISO and SIMO TR

(a)

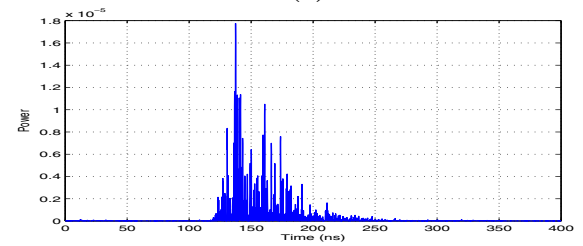

(b)

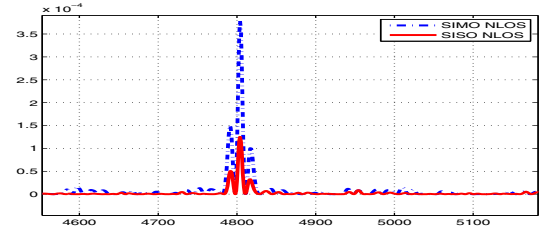

Fig. 5. In NLOS configuration a) Indoor CIR for SISO b) Indoor TR response for SISO and SIMO TR

\section{A. SIMO in Reverberation Chamber}

$\mathrm{RC}$ is a metallic chamber. It generates large amount of multipaths and is thus a perfect environment for a TR system. SISO and SIMO experiments are carried out in RC and the comparison is summarized in Table I. Fig. 3a shows power delay profile (PDP) of CIR with SISO configuration in RC. Large number of multipaths can be observed. Fig. $3 \mathrm{~b}$ shows TR response in RC for both SISO and SIMO configurations for an equal value of transmitted energy. Almost all the multipaths are coherently added and the resulting signal has very high FG and SSR. Furthermore, it can be seen that SIMO TR has a better TR peak performance compared to SISO TR. Table I presents different characteristics of SIMO and SISO TR in RC environment. It shows that FG, average power increase, SSR and TR peak performance normalized to the SISO TR system improves significantly with SIMO TR. For instance, it can be seen that TR peak with SIMO TR is $4.84 \mathrm{~dB}$ more than SISO TR for same transmitted energy. 
TABLE II

TIME REVERSAL CHARACTERISTICS IN AN INDOOR ENVIRONMENT

\begin{tabular}{ccccc}
\hline \hline \multirow{2}{*}{ TR Property } & \multicolumn{2}{c}{ LOS } & \multicolumn{2}{c}{ NLOS } \\
& SISO & SIMO & SISO & SIMO \\
\hline \hline Focusing Gain $(\mathrm{dB})$ & 9.1 & 10.8 & 11.0 & 11.5 \\
Avg. Increased Power & 3.5 & 2.7 & 3.9 & 5.2 \\
SSR & 4.3 & 4.5 & 4.0 & 4.0 \\
$d s / d s_{T R}$ & 2.1 & 2.8 & 2.3 & 2.7 \\
Normalized TR peak & 0 & 3.48 & 0 & 4.29 \\
\hline
\end{tabular}

\section{B. SIMO in an Indoor Environment}

SISO and SIMO Experiments are carried out in an indoor environment with both line of sight (LOS) and non line of sight (NLOS) configurations and the results are summarized in TableII. Figs. $4 \mathrm{a}$ and 5a show the measured SISO CIR in an indoor environment for LOS and NLOS configurations respectively. NLOS configuration has got larger number of multipaths and is thus better adopted for a TR system. Figs. $4 \mathrm{~b}$ and $5 \mathrm{~b}$ show the measured TR response for both SISO and SIMO configurations in LOS and NLOS configurations respectively. Total transmitted energy for all the experiments is kept same. Table II presents different characteristics of SIMO and SISO TR in an indoor environment for LOS and NLOS configurations. In case of LOS configuration, it is quite obvious that the TR peak performance is significantly better in the case of SIMO TR. For two receiving antennas, an improvement of $3.48 \mathrm{~dB}$ is observed. All other TR properties also improve except for average increase power. In the case of NLOS configuration, we observe even better TR peak performance. This time we achieve an improvement of 4.29 $\mathrm{dB}$ with SIMO configuration. The other factors also improve but SSR remains same. Thus SIMO NLOS system has the best TR performance amongst all systems. All TR properties improve significantly except for SSR which remains almost unchanged for both SISO and SIMO systems.

\section{ACKNOWLEDGMENT}

This work was partially supported by ANR Project MIRTEC and French Ministry of Research.

\section{CONCLUSION}

In this paper, time domain measurements are presented for a SIMO TR system in RC and in indoor environments. It is shown that SIMO TR improves different TR characteristics e.g. focusing gain, increased average power, signal to side lobe ratio, TR peak performance and RMS delay spread. In RC, SIMO and SISO TR measurements give a very high focusing gain and SSR. For instance, the focusing gain for SIMO TR in $\mathrm{RC}$ is $28.5 \mathrm{~dB}$. TR peak performance of SIMO TR is $4.84 \mathrm{~dB}$ better than peak performance of SISO TR. In indoor, SIMO TR has a lot better performance than SISO TR especially for NLOS configuration. The TR peak performance of SIMO TR is 3.48 and $4.29 \mathrm{~dB}$ better than SISO TR for LOS and NLOS configurations respectively. Thus amongst all configurations, SIMO NLOS TR system gives the best performance.

\section{REFERENCES}

[1] R. Hoctor and H. Tomlinson, "Delay-Hopped Transmitted-Reference RF Communications," Proc. IEEE 2nd Ultra Wideband Systems and Technologies (UWBST02), Baltimore, MD, pp. 265-269, May 2002.

[2] R. J. Fontana, E. Richley, and J. Barney, "Commercialization of an Ultra Wideband Precision Asset Location System," Proc. IEEE Conference UWB systems and Technologies, Reston, VA, 2003.

[3] J. D. Choi and W. E. Stark, "Performance of Ultra-Wideband Communications with Suboptimal Receivers in Multipath Channels," IEEE Journal on Selected Areas in Communications, vol. 20, pp. 1754-1766, Dec. 2002.

[4] M. Z. Win and R. A. Scholtz, "Impulse radio: How it works", IEEE Commun. Lett., vol. 2, no. 2, pp. 36-38, Feb. 1998.

[5] M. Z. Win and R. A. Scholtz, "Characterization of ultra-wide bandwidth wireless indoor channels: A communication-theoretic view ", IEEE J. Select. Areas Commun. (JSAC), vol. 20, no. 9, pp. 1613-1627.

[6] M. Fink, "Time-reversed acoustic", Scientific Amer., pp. 67-73, Nov. 1999.

[7] A. Derode, A. Tourin, J. D. Rosny, M. Tanter, S. Yon, and M.Fink, "Taking advantage of multiple scattering to communicate with timereversal antennas", Phys. Rev. Lett., vol. 90, no. 1, pp. 10143011014301-4, 2003.

[8] H. T. Nguyen,I. Z. Kovcs,P. C.F. Eggers, ”A Time Reversal Transmission Approach for Multiuser UWB Communications", IEEE Transactions On Antennas and Propagation, Vol. 54, NO. 11, November 2006.

[9] P. Kyritsi, G. Papanicolaou, P. Eggers, and A. Oprea, "MISO time reversal and delay-spread compression for FWA channels at $5 \mathrm{GHz}$ ", IEEE Antennas Wireless Propag. Lett., vol. 3, no. 6, pp. 96-99, 2004.

[10] P. Kyritsi, G. Papanicolaou, P. Eggers, and A. Oprea, "One-bit Time Reversal for WLAN Applications", Personal, Indoor and Mobile Radio Communications, 2005. PIMRC 2005.

[11] A. E. Akogun, R. C. Qiu, and N. Guo, "Demonstrating time reversal in ultra-wideband communications using time domain measurements," in 51st Int. Instrument. Symp., Knoxville, TN, May 8-12, 2005.

[12] C. Oestges, J. Hansen, S. M. Emami, , A. D. Kim, G. Papanicolaou, A. J. Paulraj, "Time Reversal Techniques for Broadband Wireless. Communication Systems." European Microwave Conference (Workshop), Amsterdam, The Netherlands, October 2004, 2004, 49-66.

[13] H. T. Nguyen, J. B. Andersen, and G. F. Pedersen, "The potential use of time reversal technique in multiple elements antenna systems", IEEE Commun. Lett., vol. 9, no. 1, pp. 40-42, Jan. 2005.

[14] T. Strohmer, M. Emami, J. Hansen, G. Papanicolaou, and P. J. Arogyaswami, "Application of time-reversal with MMSE equalizer to UWB communications", in Proc. IEEE Global Telecommunications Conf. (GlobeCom), Dec. 2004, vol. 5, pp. 3123-3127.

[15] R. C. Qiu, C. Zhou, N. Guo, and J. Q. Zhang, "Time Reversal with MISO for Ultra-Wideband Communications: Experimental Results", IEEE Antenna and Wireless Propagation Letters, 2006.

[16] A. Khaleghi, G. El Zein, I. H. Naqvi, Demonstration of Time-Reversal in Indoor Ultra-Wideband Communication: Time Domain Measurement, ISWCS 2007, Trondheim, Norway.

[17] A. Khaleghi, G. El Zein, "Signal Frequency And Bandwidth Effects On The Performance Of UWB Time-Reversal Technique", Loughborough Antennas and Propagation Conference 2007, Loughborough, UK.

[18] I. H. Naqvi, A. Khaleghi, G. El Zein "Performance Enhancement of a Multiuser Time Reversal UWB Communication System", IEEE International Symposium on Wireless Communication Systems 2007, Trondheim, Norway, accepted for publication. 